\title{
Archnet 2.0: Building a new asset management system for MIT's online architectural library of the Islamic World
}

Andrea Schuler is the Visual Resources Librarian for Islamic Architecture in the Aga Khan Documentation Center (AKDC) at MIT. In that role she manages the creation, cataloging, uploading and storage of digital assets from the AKDC's vast collections. She administers the Aga Khan Visual Archive and provides access to the Documentation Center's visual collections for the MIT community and for a worldwide community of users. She also contributes content to Archnet as part of the AKDC team.

\begin{abstract}
Archnet 2.0 is a new front end website and back end cataloging tool and asset management system for Archnet (http://archnet.org), the largest openly accessible online architectural library focusing on the Muslim world. The site, a partnership between the Massachusetts Institute for Technology and the Aga Khan Trust for Culture (AKTC), contains articles and publications, images, maps and drawings, video, archival material, course syllabi, and site records relating to architecture in the Islamic world. By 2012, when the Aga Khan Documentation Center (AKDC) at MIT, part of the MIT Libraries, took over the digital library, the site has become dated and was no longer meeting the needs of its users. A software development firm was hired to build new front and back ends for the site. This paper explores the development of the new back end cataloging and asset management tool, including the reasoning behind building a new tool from scratch; considerations during development such as database structure, fields, and migration; highlights of features; and lessons learned from the process.
\end{abstract}

Keywords: digital libraries, digital asset management, cataloging, Islamic architecture, open source, media

\section{Introduction to Archnet}

Archnet (http://archnet.org) is "a globally-accessible, intellectual resource focused on architecture, urbanism, environmental and landscape design, visual culture, and conservation issues related to the Muslim world." ${ }^{1}$ The site was officially launched in 2002 as a partnership between the Massachusetts Institute of Technology (MIT) and the Aga Khan Trust for Culture (AKTC), and has since become the largest openly accessible online architectural library focusing on the Muslim world, with a mission to provide unique visual and textual content to further teaching, scholarship, and practice.

Over the past twelve years, the digital library has been built with content contributions from the Aga Khan Trust for Culture in Geneva, the Aga Khan Programs in Islamic Architecture at MIT and Harvard, and donations from scholars, architects, and institutions from around the world. The site focuses on the Islamic world, but the content is broad and material is available relating to sites in 95 different countries. Content types available on the site include articles and publications, images, maps and drawings, video, a growing collection of archival material, course syllabi and site records. Currently, as of July 2014, the library contains nearly 8,000 articles and publications, 86,000 images, 7,000 site records, and 171 unique collections.

In January 2012, the Aga Khan Documentation Center (AKDC) at MIT, part of the MIT Libraries, took over management of the Archnet Digital Library. At this point, Archnet was 10 years old and the site was no

Journal of Digital Media Management Vol. 3, 2, 109-116 (C) Henry Stewart Publications 2047-1300 (2014).

http://www.ingentaconnect.com/content/hsp/jdmm/2014/00000003/00000002/art00004 
longer meeting the scholarly or technological needs of its users. The Trust for Culture hired a software development firm to begin work on an entirely new front end site and back end cataloging tool and asset management system for Archnet, built from the ground up. This site would ultimately go live to the world on January 27, 2014.

There was no question that a new front end for Archnet was needed. The old front end was dated, not visually pleasing, and consisted primarily of long lists of content and search results. For a library that contains so much visual content, very little of that came through on the front end, and it was difficult to browse search results or collections of objects. This was becoming increasingly important as the volume of content on the site grew. Furthermore, the site lacked the ability to package and present content in learning and teaching tools.

The reasoning for building an entirely new back end tool from scratch was more complex. Archnet had an existing Filemaker Pro back end database, custom-built in the early 2000s. Because of its age and convoluted data structure, and the suite of new functionality needed, modifying this tool to meet current needs was never an option that was seriously considered. Using another existing cataloging tool was considered, including ARTstor's Shared Shelf media management software ${ }^{2}$ or traditional visual resources tools in use at MIT, Harvard, and other institutions. However, after exploring those options, it became apparent that the unique combination of material in Archnet, coupled with desired current and future functionality, meant that no existing tool would meet the site's needs.

\section{Unique content functionality}

Nearly 7,000 site records sit at the heart of the Archnet site, setting it apart from other digital libraries. These site records package together descriptive text about a building, monument, or restoration project, a bibliography, geographic location information, and related media including images, articles and publications, drawings, and video. A back end system would have to accommodate that structure and have the ability to associate freely between objects of different types, including the various media types, name authority records, collections, and parent records to child records. Figure 1 shows an example of an Archnet site record on the front end, with its many types of associations.

Additionally, many of the existing cataloging and asset management tools that were considered in the early phases of the new Archnet development were largely built for one media type. Traditional visual resources databases, for example, could handle images well but not the large body of articles and publications found in Archnet. Archival databases would work well for the growing collection of architect's archives in Archnet, but would not accommodate images or videos as easily. There was truly no one-size-fits-all solution available that would work.

Journal of Digital Media Management Vol. 3, 2, 109-116 (C) Henry Stewart Publications 2047-1300 (2014). http://www.ingentaconnect.com/content/hsp/jdmm/2014/00000003/00000002/art00004 


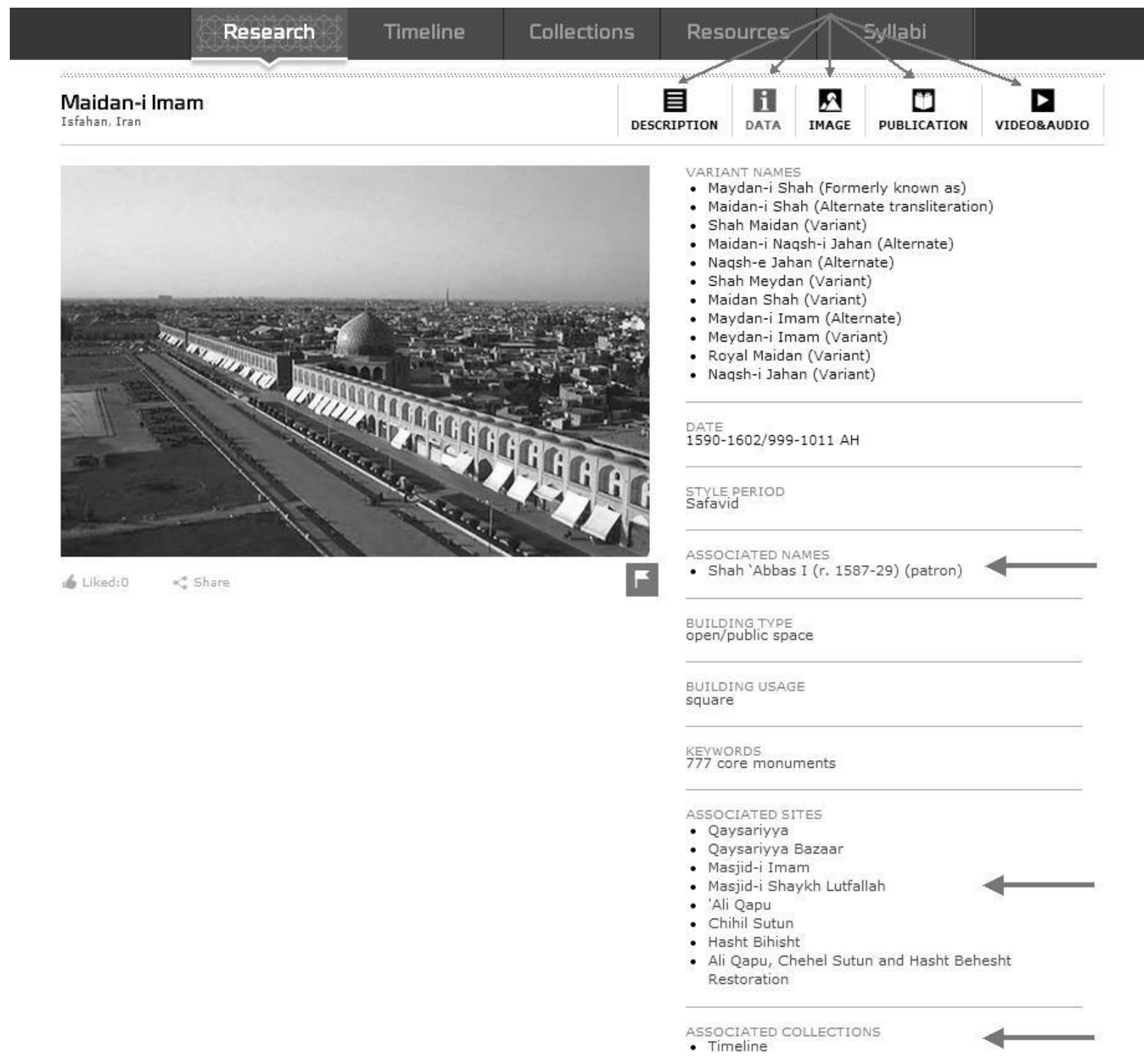

\section{Development}

Development on the new site, both front and back end, began in 2011, before Archnet was even officially handed over to the Libraries. Due to the budget, work was suspended for nearly all of 2012 before picking up again in early 2013. The new back end tool was available for staff to use beginning in November 2013, and the new front end site went live to the world in January 2014. The software develop firm retained by the Trust for Culture did all of the actual development work, while in constant contact with MIT Libraries and AKTC staff for input on functionality, content, usability, and standards. A number of on-site meetings were held, conference calls were frequently scheduled, and wireframes and specifications were repeatedly exchanged, commented upon, and implemented.

Journal of Digital Media Management Vol. 3, 2, 109-116 (C) Henry Stewart Publications 2047-1300 (2014).

http://www.ingentaconnect.com/content/hsp/jdmm/2014/00000003/00000002/art00004 
A suite of open source tools were used in the development of the new site. These include:

- Development

- Ruby (high level programming language)

- Git (Distributed Version Control system)

- Frameworks and libraries on back end (server-side)

- Ruby on Rails (framework for creating web applications)

- Capistrano (deployment manager for Ruby applications)

- Puma (a Ruby webserver)

- Frameworks on front-end (client-side)

- jQuery (useful library for HTML Document manipulations, event handling and AJAX calls)

- Bootstrap (front-end framework for web development)

- Backbone.JS (structure for client-side web application)

- $\quad$ Project Hydra

- Jetty (servlet engine - lightweight web server for Java-based application, used by Project Hydra)

- Solr (search engine, with ability to full-text search, used by Project Hydra for fast data retrieval)

- Fedora Repository

- Project Hydra (repository solution based on Fedora Repository and Solr)

- Server

- Ubuntu Server LTS (popular Linux distribution)

- Nginx (HTTP and reverse proxy server, used as front-end server sending static files from disk to users (image thumbnails, compiled CSS and JS files) and forwarding application request to back end Puma server)

- PostgreSQL (relational database engine)

The architecture behind the site was determined by the development team, but much work had to be done by AKDC staff in order to determine database fields, structure, and mapping from the old database. The old Filemaker database provided the backbone for the new database, and additional fields were added to provide more granularity and to accommodate cataloging of additional material types that were less of a focus for the library when the site original launched. Major changes included the addition of repeating fields (the site name field, for example, previously listed all the variant names in one long comma-separated string. The new site allows each to be in its own field); additional fields for the description of original visual material found in archival collections; keyword fields; and dropdown menus for agent roles (we can now clarify if the name assigned to a site record is, for example, the architect, the patron, or the restoration architect).

Because of the wide variety of material included in the Archnet library, a variety of data and content standards were consulted when selecting fields for the cataloging tool. The selection of fields for several tables, including images, sites, and authorities, was informed by the data standard VRA Core $4.0^{3}$, with some necessary modifications. Drop-down lists in the database for elements such as agent role, material type, media type, and keywords were populated with values from authorities including Getty's Art \& Architecture Thesaurus ${ }^{4}$ (AAT) and Library of Congress subject headings $s^{5}$ (LCSH). For textual material, citation guidelines based on the Chicago Manual of Style were drawn up. Other content standards

Journal of Digital Media Management Vol. 3, 2, 109-116 (C) Henry Stewart Publications 2047-1300 (2014). 
consulted include Cataloging Cultural Objects $^{6}$ (CCO) and Describing Archives: A Content Standard (DACS).

One aspect of the development that was particularly nerve-wracking was the migration of data from the old Filemaker database into the new back end tool, as there seemed to be so many possibilities for data to end up in the wrong place, or to become illegible. In fact, thanks to careful planning by the developers and by the AKDC team before the import, this step ran relatively smoothly. Once a confirmed list of fields in the new database was finalized, the AKDC team mapped those fields to the existing fields in the Filemaker database. For any new fields, the team determined if a default value should be entered during the migration, or if it should be left blank. For example, as mentioned above, one new field in the new back end was "agent role." In the old database, names of architects, planners, or other people and firms associated with a site had no role assigned to them. In the new database, the role of "architect/planner" was assigned as a placeholder value for agent role for all names, and will be edited to be more specific as the team works to clean up and edit records in the new database. Other new fields, such as media dimensions, were left blank, as there was no appropriate default value.

Field delimiters needed to be determined for any fields that were previously long strings of many values but that were being broken out into repeating fields in the database, and a good deal of time was spent cleaning up these fields to ensure that the data would transfer cleanly. Even with this cleanup, there was of course still cleanup necessary after the migration. For example, in some fields a comma was selected as a delimiter between multiple values in a string, since there should not have been commas anywhere in the terms themselves. However, after the migration it was discovered that some terms had been improperly entered with commas in the old database, and thus had been split improperly into the new database. Because of the sheer volume of data in the database it was not possible to catch of these things before migration, or even after, but they are being cleaned up as issues are encountered.

\section{Back end features}

The overall structure of the new database remains similar to the old structure. There are tables for cataloging sites, images \& video, and documents, as well as for creating a name authority file, a donor file, and a donation file. All of these tables were populated with values from the old Filemaker database. The development of the new back end tool, however, offered the opportunity to gain new fields, features, and functionality that had been missing from the previous database, a few of which will be highlighted here.

One of the largest changes was the decision to build a web-based tool. This was particularly important for Archnet, as the site works with contributors from around the globe and a web tool would facilitate moving towards a model where remote scholars and researchers can enter material directly into the database without having to be located in Cambridge, or without having to download software and connect to a server. It also allows staff the flexibility to work while traveling or while on-site at another institution. All of the assets - images, articles, videos, etc. - are stored directly within the database in the new version, rather than sitting separately on a server as in the previous tool. This makes cataloging simpler, as the object being cataloged is sitting directly in the cataloging screen, and makes it easy to tell at a glance if a record has a missing, incorrect, or corrupt file attached.

Journal of Digital Media Management Vol. 3, 2, 109-116 @ Henry Stewart Publications 2047-1300 (2014).

http://www.ingentaconnect.com/content/hsp/jdmm/2014/00000003/00000002/art00004 
The new back end tool was created with the goal of a cleaner data structure, allowing the data to more easily be shared, manipulated, or migrated in the future. As mentioned earlier, the structure of many fields in the database was changed from a single box with many comma-separated values within to repeating fields with one piece of data per field. New qualifier fields help to make this data more precise and legible.

One seemingly small change, but one that has actually played a large role in front end browsability and display of data and search results, was the addition of cover image functionality. This allows staff to upload a "cover" image with any asset, giving the ability, for example, to have a journal issue or article display with an image of the journal cover, or a collection to have a key image display, or to have a photo of an architect display with his/her name record.

Furthermore, the new back end tool built in functionality to easily reorder the images associated with a site record, and to change the way they display on the front end. This is significant, because for architectural sites, there is a logical order that images should appear to most effectively represent the site - for example, historic images should appear before contemporary images and exterior images should appear before interior images. In the old tool, images displayed simply in the order that they were uploaded to the server. If an additional image was later added to the record, it would be tacked on at the end, and if the cataloger wanted it to appear at a different point in the list of images, every single image would have to be re-cataloged in that order and re-uploaded. In the new tool, a cataloger can view a grid display of all the images associated with a site record, and drag and drop them into the desired order, as well as set the main image that displays for the site record.

One of the most important features in the new back end tool is the ability to create collections, which subsequently display to users on the front end, and to have objects of all types associated with them. In the old site, there could be a collection of images, and a collection of publications, but there could not be a collection with both images and publications in it. Especially for archival collections, which are making up a growing percentage of Archnet's content, this simply did not work. Staff needed the ability to create collections that grouped objects of all types together: for example, in a collection related to the Iraqi architect Mohamed Makiya, whose archive the Aga Khan Documentation Center at MIT acquired in 2012, there are name authority records, images, publications, site records, and videos. In the old site, this material would not have been able to be associated together and users would have had to browse for it in multiple locations.

The new tool also allows geographic coordinates to be set for site records and for images, adding an additional layer of data and specificity to the records. Catalogers can manually enter the coordinates, or choose the location from an inset Bing Maps map that appears directly in the cataloging tool. This then displays to users on the front end. Other functionality added to make the cataloging process easier includes the ability to globally copy and paste record metadata into another record, import it in with a CSV file, and bulk upload images into the database. A text editor is built directly into free-text description and citation fields, providing much more control over how the text is formatted and not requiring staff to use HTML markup as the old tool did.

Finally, back end tools were also built to facilitate the development of front end teaching and learning tools, such as an interactive timeline with key dynasties and sites over the history of Islamic

Journal of Digital Media Management Vol. 3, 2, 109-116 (C) Henry Stewart Publications 2047-1300 (2014).

http://www.ingentaconnect.com/content/hsp/jdmm/2014/00000003/00000002/art00004 
architecture; a "walk-through" of an Aga Khan Historic Cities-rehabilitated park in Cairo that serves as a portal to other Historic Cities material; and a portal to all of the cycles of the Aga Khan Award for Architecture. As with collections, objects are associated with each of these tools in the back end and packaged together for users on the front end.

\section{Lessons Learned}

The development of Archnet 2.0 was a learning experience for everyone on the AKDC staff, and the team came away with a number of important lessons learned that can inform future development projects both for our staff and for others considering taking on a similar project. These include:

- A project like this will take longer than expected. If there is a hard deadline, build extra time into the project, and if there is a soft deadline, accept that it will likely get pushed back. Unexpected issues, both small and mundane or potentially large and serious are going to crop up.

- When working with an outside consultant, look for one that has experience with the kind of tool that's being built. This will save time, bring knowledge and experience to the process, and ultimately cut down on the frustration of the client and the development team not "speaking the same language."

- Research what other tools are available, what organizations are doing similar work, and who might be available to work with. Don't build in a vacuum, and don't reinvent the wheel.

- Perhaps most importantly, perform a thorough assessment of all the desirable features in the system currently in use, if there is one, and all of the desirable features found in other systems or otherwise wanted for the new system, and write them all down. Do this no matter how small the item seems. There was some functionality that our team took for granted, or that was so small it wasn't mentioned, and that functionality inadvertently got left out of the final product. If possible, have the people who are building the site sit down with the staff who will be using it, watch them work for a while, and see what works and what doesn't work in the current workflow.

- In communication with the developers, write everything down. As in the item above, document it all, no matter how seemingly small. It can then be referred back to if necessary. The team at the development firm working on this project changed at least twice over the course of the project term, and a good deal of knowledge was lost in those transfers. Having notes, documents, and emails to refer back to was essential.

- Make certain that the tool that's being designed works for its intended user base. Don't allow unnecessary features because someone else thinks they're cool or thinks that's how it should be done. Everything needs to be suited to the workflow of the users who will be using the tool day in and day out, and unnecessary fields and features are a distraction and cause confusion later on.

- Even after a successful launch, things will need to be changed. User testing can mitigate some of this, but there will inevitably be things omitted, or that do not work as expected. For the Archnet back end, examples of changes made post-launch include changes to the display to make it more readable on a single screen without scrolling; the ability to edit and add to dropdown lists that had been populated from the previous database but made static; and the addition of an advanced search with more granularity to search specific fields and build complex

Journal of Digital Media Management Vol. 3, 2, 109-116 (C) Henry Stewart Publications 2047-1300 (2014). 
queries, mimicking the improved searchability on the front end. Additionally, numerous bug fixes were put into place as they cropped up.

Ultimately, the team's deep understanding of Archnet content and the necessary functions of a new tool coupled with careful planning and development resulted in a new tool that continues to serve both back end and front end users well. We expect both the back end and the front end to continue to evolve over time, perpetually working towards the mission of making Archnet's content open and accessible to a worldwide audience to further teaching, scholarship, and practice.

${ }^{1}$ Archnet. 'Archnet - About', available at http://archnet.org/pages/about (accessed 22 July 2014).

${ }^{2}$ ARTstor 'Shared Shelf Overview,' available at http://www.artstor.org/shared-shelf/s-html/shared-shelfhome.shtml (accessed 22 July 2014).

${ }^{3}$ Visual Resources Association 'VRA CORE,' available at http://www.loc.gov/standards/vracore/ (accessed 24 July 2014).

${ }^{4}$ Getty Research Institute 'Art \& Architecture Thesaurus,' available at http://getty.edu/research/tools/vocabularies/aat/index.html (accessed 24 July 2014).

${ }^{5}$ Library of Congress 'Library of Congress Authorities,' available http://authorities.loc.gov/ (accessed 24 July 2014).

${ }^{6}$ Visual Resources Association 'CCO Commons," available at http://cco.vrafoundation.org/ (accessed 24 July 2014).

Journal of Digital Media Management Vol. 3, 2, 109-116 (C) Henry Stewart Publications 2047-1300 (2014). http://www.ingentaconnect.com/content/hsp/jdmm/2014/00000003/00000002/art00004 